\title{
Various MRS Application Tools for Alzheimer Disease and Mild Cognitive Impairment
}

F. Gao and P.B. Barker

\begin{abstract}
SUMMARY: MR spectroscopy is a noninvasive technique that allows the detection of several naturally occurring compounds (metabolites) from well-defined regions of interest within the human brain. Alzheimer disease, a progressive neurodegenerative disorder, is the most common cause of dementia in the elderly. During the past 20 years, multiple studies have been performed on MR spectroscopy in patients with both mild cognitive impairment and Alzheimer disease. Generally, MR spectroscopy studies have found decreased Nacetylaspartate and increased myo-inositol in both patients with mild cognitive impairment and Alzheimer disease, with greater changes in Alzheimer disease than in mild cognitive impairment. This review summarizes the information content of proton brain MR spectroscopy and its related technical aspects, as well as applications of MR spectroscopy to mild cognitive impairment and Alzheimer disease. While MR spectroscopy may have some value in the differential diagnosis of dementias and assessing prognosis, more likely its role in the near future will be predominantly as a tool for monitoring disease response or progression in treatment trials. More work is needed to evaluate the role of MR spectroscopy as a biomarker in Alzheimer disease and its relationship to other imaging modalities.
\end{abstract}

ABBREVIATIONS: AD = Alzheimer disease; GABA $=\gamma$-aminobutyric acid; Glu $=$ glutamate; ${ }^{1} \mathrm{H}$-MR spectroscopy $=$ proton $\mathrm{MR}$ spectroscopy; $\mathrm{LASER}=$ localization by adiabatic selective refocusing; $\mathrm{MCl}=$ mild cognitive impairment; $\mathrm{ml}=$ myo-inositol; $\mathrm{MRSI}=\mathrm{MR}$ spectroscopic imaging; PRESS = point-resolved spectroscopy sequence; SENSE = sensitivity encoded; $\mathrm{tCr}=$ total creatine; $\mathrm{tNAA}=$ total $\mathrm{N}$-acetyl aspartate; $\mathrm{TMA}=$ trimethylamines

M $\mathrm{R}$ spectroscopy is a noninvasive technique that permits the estimation of the concentrations of various compounds (metabolites) in the human brain in vivo. To be detectable by in vivo MR spectroscopy, a compound must be a relatively small molecule present in at least the low-millimolar concentration range. MR spectroscopy may be performed by using the signals from a number of different nuclei, including phosphorus $\left({ }^{31} \mathrm{P}\right)$, carbon $\left({ }^{13} \mathrm{C}\right)$, and fluorine $\left({ }^{19} \mathrm{~F}\right),{ }^{1}$ but ${ }^{1} \mathrm{H}$-MR spectroscopy (proton MR spectroscopy) has become the most prevalent since the early 1990s because of its higher signal sensitivity, better spatial resolution, and the fact that (unlike other nuclei) no special hardware beyond that found on standard MR imaging scanners is required. ${ }^{2}$ During the past 2 decades, numerous studies have used

Received November 3, 2013; accepted after revision February 26, 2014.

From the Shandong Medical Imaging Research Institute (F.G.), Shandong University, Jinan, China; Russell H. Morgan Department of Radiology and Radiological Science (P.B.B.), Johns Hopkins University School of Medicine, Baltimore, Maryland; and F.M. Kirby Research Center for Functional Brain Imaging (P.B.B.), Kennedy Krieger Institute, Baltimore, Maryland.

This work was supported, in part, by National Institutes of Health grant P41EB015909.

Please address correspondence to Peter B. Barker, PhD, Department of Radiology, Park 367B, Johns Hopkins University School of Medicine, 600 N Wolfe St, Baltimore, MD 21287; e-mail: pbarker2@jhmi.edu

- Indicates open access to non-subscribers at www.ajnr.org

http://dx.doi.org/10.3174/ajnr.A3944
${ }^{1} \mathrm{H}-\mathrm{MR}$ spectroscopy to study both Alzheimer disease (AD) and mild cognitive impairment (MCI). This article reviews the information content of ${ }^{1} \mathrm{H}-\mathrm{MR}$ brain spectra, gives an update on the current methods used to collect MR spectroscopy (and MR spectroscopic imaging [MRSI]) data, and describes the results of MR spectroscopy research studies in patients with MCI and AD.

\section{Brain Metabolites Detectable by In Vivo MR Spectroscopy} Various metabolites are detectable at 1.5 or $3 \mathrm{~T}$ with ${ }^{1} \mathrm{H}-\mathrm{MR}$ spectroscopy in normal brain, including the prominent resonances of total NAA (tNAA), trimethylamines (TMA), total creatine (tCr), and signals from myo-inositol (mI), glutamate (Glu), and glutamine. Lactate is not usually seen in normal brain but is detectable in pathologies that cause its concentration to increase. Using special techniques (ie, spectral editing), one can detect other compounds such as $\gamma$-aminobutyric acid (GABA) and glutathione. The following is a description of the metabolites that have been the main interest to date in studies of $\mathrm{AD}$ and $\mathrm{MCI}$ :

tNAA (2.01-ppm singlet), which is estimated to have a healthy adult brain concentration on the order of $10-12 \mathrm{mmol} / \mathrm{L}$, is usually the largest signal in the spectrum. ${ }^{3}$ NAA is an amino acid synthesized in neuronal mitochondria from aspartate and acetylcoA and is primarily seen in neurons, axons, and dendrites within the central nervous system. ${ }^{4}$ Therefore, NAA is commonly referred to as a "neuronal marker," indicative of neuronal density 
and viability. However, some in vitro studies have shown that NAA can also be found in immature oligodendrocytes and astrocyte progenitor cells. ${ }^{5}$ Furthermore, some longitudinal studies suggested that NAA undergoes reversible changes in patients, suggesting it can vary for reasons other than neuronal density. ${ }^{6,7}$ Overall, the balance of evidence suggests that NAA is present predominantly in neurons and that it is a reasonably good surrogate marker of neuronal health in many neurologic and psychiatric disorders. The peak detected by MR spectroscopy also contains a small contribution from $\mathrm{N}$-acetyl aspartylglutamate, so for this reason, it is sometimes referred to as total $\mathrm{N}$-acetyl aspartate (tNAA). In addition, in certain rare diseases, it is thought that the tNAA peak may also contain contributions from $\mathrm{N}$-acetyl neuraminic acid (sialic acid) and uridine-diphosphate- $N$-acetyl-sugars. ${ }^{8}$

TMA (3.20-ppm singlet) are a composite peak predominantly containing contributions from glycerophosphorylcholine (a product of the breakdown of membrane phosphatidylcholine) and phosphocholine (a precursor of phosphatidylcholine). There may also be small contributions from free choline, acetylcholine, carnitine, and acetyl-L-carnitine. Since phosphocholine and glycerophosphorylcholine are involved in both the synthetic and degradation pathways of cell membranes, the amplitude of the TMA peak is sometimes thought to be related to membrane turnover. ${ }^{9}$ In addition, it has been shown that glial cells have high levels of TMA. $^{10}$

tCr (3.01-ppm singlet, 3.9-ppm singlet) consists of the sum of creatine and phosphocreatine, which are energy metabolites. $\mathrm{tCr}$ is frequently used as an "internal reference" to quantify other neurochemicals. However, in normal brain, lower levels of $\mathrm{tCr}$ are found in white matter than in gray matter, and higher levels of $\mathrm{tCr}$ are found in the cerebellum compared with supratentorial regions. Some studies have suggested that $\mathrm{tCr}$ levels increase slowly with age. ${ }^{11}$ Therefore, the assumption of constant $\mathrm{tCr}$ when used as an internal reference should be viewed with caution.

$\mathrm{mI}$ (3.3-, 3.5-, and 4.0-ppm multiplets) is a sugar alcohol containing contributions from free $\mathrm{mI}$ and $\mathrm{mI}$ phosphate. ${ }^{12} \mathrm{mI}$ is readily detectable in short (eg, $\leq 35 \mathrm{~ms}$ ) TE spectra; however, it is usually not detectable at long TEs. Because $\mathrm{mI}$ has been suggested to be higher in glial cells than in neurons, ${ }^{13,14} \mathrm{mI}$ is often considered as a "glial cell marker." $\mathrm{mI}$ also may act as a marker of a detoxification agent or it may be an osmoregulator or intracellular messenger. ${ }^{12,13,15}$

Glu and glutamine (2.1-2.4- and 3.7-ppm multiplets) structures are complex; hence, their peaks are difficult to separate, both from each other and from other compounds. Therefore, they are frequently detected as a composite "Glx" peak. ${ }^{16}$ Glu is the major excitatory neurotransmitter in the brain, and glutamine may play a role in detoxification and regulation of its precursor, Glu, within the astrocyte body. ${ }^{12}$ It has been suggested that the Glu signal in brain ${ }^{1} \mathrm{H}-\mathrm{MR}$ spectroscopy primarily reflects the intracellular compartment.

GABA (1.9-, 2.3-, and 3.0-ppm multiplets) is the primary inhibitory neurotransmitter in the brain. ${ }^{17}$ However, it is difficult to detect GABA by using conventional ${ }^{1} \mathrm{H}$-MR spectroscopy because resonances of GABA overlap with other metabolites such as $\mathrm{tCr}$, Glu, tNAA, and macromolecules. In addition, normal brain
GABA concentrations are at relatively low levels $(1.3-1.9 \mathrm{mmol} /$ $\left.\mathrm{kg}_{\text {wet weight }}\right) .{ }^{18}$ For these reasons, most MR spectroscopy studies to date have used spectral-editing methodologies to reliably detect GABA free from contamination from larger overlapping signals.

Methods for In Vivo MR Spectroscopy in the Human Brain Spatial localization methods for ${ }^{1} \mathrm{H}-\mathrm{MR}$ spectroscopy consist of single-voxel techniques, in which spectral data are acquired from 1 location at a time, or multivoxel techniques. In multivoxel spectroscopy, referred to as MRSI or chemical shift imaging, spectra from multiple regions are acquired simultaneously.

Single-Voxel Techniques. The 2 most commonly used singlevoxel MR spectroscopy techniques are known as the pointresolved spectroscopy sequence (PRESS) ${ }^{19}$ and STEAM. ${ }^{20}$ Both techniques use 3 mutually orthogonal section-selective pulses and design the pulse sequence to collect only an echo signal from the point (voxel) in space where all 3 sections intersect. In the STEAM sequence, three $90^{\circ}$ pulses are used to provide a so-called "stimulated echo," whereas in the PRESS sequence, one $90^{\circ}$ and two $180^{\circ}$ refocusing pulses are applied to create a spin-echo. STEAM and PRESS are generally similar but differ in some respects. The biggest difference is that the PRESS should have approximately a factor-of-2 better signal-to-noise ratio than STEAM and is therefore commonly used. However, it is possible to achieve shorter TE values in STEAM than PRESS (which is important for detecting metabolites with short T2 relaxation times), and the $90^{\circ}$ sectionselective pulses in STEAM give better voxel profiles compared with the $180^{\circ}$ section-selective refocusing pulses used in PRESS. In addition, STEAM may be particularly useful for high-field brain MR spectroscopy. Figure 1 shows both short and long TE spectra recorded using the PRESS and STEAM sequences in 2 patients with $\mathrm{AD}$.

Although widely used, STEAM and PRESS do have some limitations, particularly when used at high magnetic field strengths (eg, 3T or higher). Due to wavelength effects in volume radiofrequency coils ${ }^{21}$ or when using inhomogeneous surface coils for excitation, it may be difficult to obtain uniform radiofrequency transmit $\left(B_{1}\right)$ fields. This then results in misadjustment of flip angles and variation of the flip angles within the voxel, resulting in suboptimal excitation. The localization by adiabatic-selective refocusing (LASER) sequence ${ }^{22}$ or its modified version "semiLASER," has been applied to solve these problems. ${ }^{23,24}$ The LASER sequence is a modification of the PRESS sequence by using adiabatic (usually hyperbolic secant) refocusing pulses. It consists of a non-section-selective adiabatic half-passage $90^{\circ}$ pulse for excitation, and 3 pairs of hyperbolic secant refocusing pulses in 3 directions for localization. Compared with STEAM or PRESS, a more uniform excitation profile and decreased chemical shift displacement errors may be achieved in the LASER sequence. ${ }^{25}$ However, compared with the PRESS sequence, the radiofrequency power deposition is higher and the minimum TE is longer, due to the large number of radiofrequency pulses. The semiLASER sequence consists of a nonadiabatic $90^{\circ}$ section-selective pulse and 2 pairs of adiabatic hyperbolic secant pulses for refocusing. Semi-LASER has lower radiofrequency power deposition and shorter TEs than LASER, but some insensitivity to $B_{1}$ inhomogeneity is lost. ${ }^{25}$

AJNR Am J Neuroradiol 35:S4 Supplement 2014 www.ajnr.org 

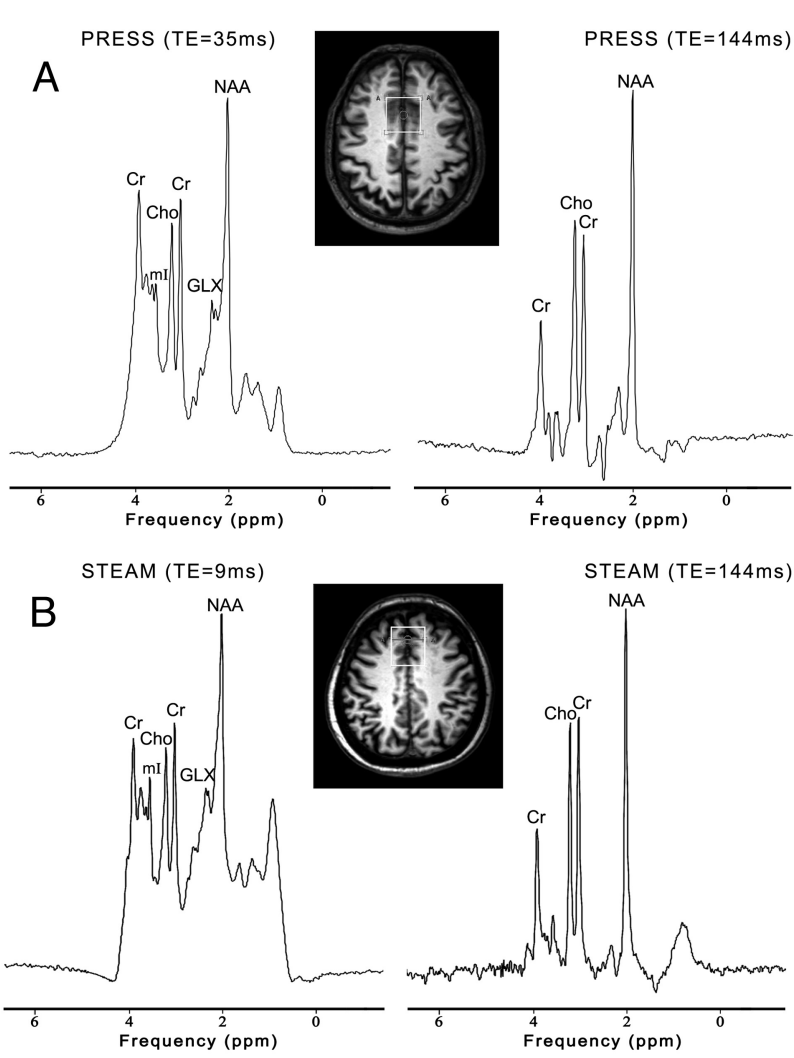

FIG 1. 3T MR spectroscopy in 2 patients with AD. A, PRESS (TR, 2000 $\mathrm{ms}$; TE, 35 and $144 \mathrm{~ms}$ ) in a 71-year-old man. B, STEAM (TR, $2000 \mathrm{~ms}$; TE, 9 and $144 \mathrm{~ms})$ in a 57-year-old man. The white box represents the location of the VOI $\left(3 \times 3 \times 3 \mathrm{~cm}^{3}\right)$ in the mesial frontal region. The lower SNR of the STEAM spectra is apparent.

The "SPECIAL" consists of a section-selective inversion pulse followed by a spin-echo sequence, with each pulse applied in a different direction. ${ }^{26}$ The sequence combines the short TE achievable with STEAM with the full signal intensity provided by PRESS. The sequence collects full-intensity signal from a column defined by the intersection of the selected sections of the $90^{\circ}$ and $180^{\circ}$ pulses. ${ }^{25}$ The section-selective inversion pulse is applied to every other TR, so that a minimum of 2 scans is required to achieve full spatial localization. ${ }^{25}$ The main limitation of spin-echo full-intensity acquired localized spectroscopy is that it is not a "singleshot" localization method and is, therefore, potentially susceptible to head motion or other instabilities, possibly leading to localization errors.

Multiple-Voxel (Spectroscopic Imaging) Techniques. Singlevoxel MR spectroscopy is readily available on nearly all MR imaging scanners, is rapid and relatively easy to perform, and has been used in most AD studies to date. However, single-voxel MR spectroscopy studies are very limited in terms of both coverage and spatial resolution. For instance, single-voxel MR spectroscopy studies of the brain are often limited to 1 or 2 regions and therefore cannot assess the spatial distribution of metabolites. In contrast, MRSI can provide an assessment of the spatial distribution of the various metabolites in addition to their relative concentrations within a voxel.

MRSI can be acquired in $2 \mathrm{D}^{27,28}$ or $3 \mathrm{D}$ and generally offers superior spatial resolution $\left(<1 \mathrm{~cm}^{3}\right)$ compared with single-voxel

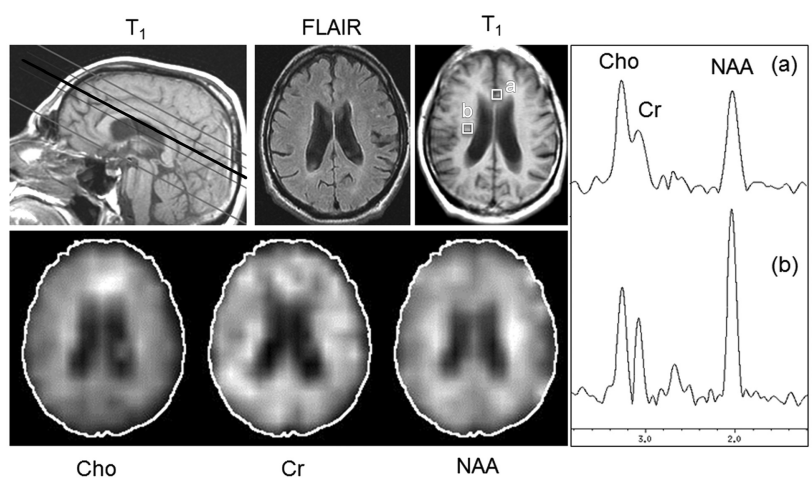

FIG 2. Long TE multisection MRSI (TR, 2300 ms; TE; 280 ms; 15-mm section thickness; $32 \times 32$ in-plane resolution; 4 sections; $1.5 T$ ) in a 72-year-old man with a diagnosis of $\mathrm{MCl}$. Metabolic images from the third of 4 sections show mildly reduced NAA in a diffuse distribution, with relative preservation of NAA bilaterally in the corticospinal tracts. Cho is mildly increased in the mesial frontal regions compared with other brain regions. Selected spectra from the anterior cingulate (a) and corticospinal tract (b) show the relative amplitudes of the Cho, $\mathrm{Cr}$, and NAA signals in these regions.

spectroscopy. An example of 1 section from a multisection 2D MRSI dataset from a patient with MCI is shown in Fig 2. Generally, ${ }^{1} \mathrm{H}$-MRSI is acquired from a volume that is prelocalized by using either PRESS or STEAM; however, as in the example in Fig 2 , it is also possible to acquire ${ }^{1} \mathrm{H}-\mathrm{MRSI}$ with only section (or 3D slab) localization. 2D or 3D MRSI with conventional phase-encoding techniques involves long acquisition times, particularly if high spatial resolution and coverage are desired. Therefore, a number of approaches for fast MRSI have been developed, such as echo-planar spectroscopic imaging, spiral MRSI, and sensitivity encoded (SENSE) MRSI.

Echo-planar spectroscopic imaging is one of the fastest acquisition techniques for MRSI. An oscillating read gradient is applied during data readout with each data point in the time-domain corresponding to a bipolar lobe of the read gradient, thus both spectral and spatial information can be collected simultaneously. ${ }^{29}$ The oscillating read gradient can be viewed as repeatedly collecting 1 line of $k$-space at different time points. Conventional phase-encoding is then applied in the other 1 or 2 directions to extend the experiment to either 2 or 3 spatial dimensions, respectively. The echo-planar spectroscopic imaging readout reduces the number of phase-encoding steps by an order of magnitude compared with conventional MRSI, thereby achieving a large scan time reduction. Echo-planar spectroscopic imaging encoding can be implemented with slab-, multisection-, or PRESS-based excitation. ${ }^{30}$ As an alternative to echo-planar spectroscopic imaging, spiral-encoded MRSI also applies a field gradient during data acquisition, but unlike echo-planar spectroscopic imaging, it traverses $k$-space in an in-to-out spiral pattern. ${ }^{31}$ Spiral-encoded MRSI has a number of potential advantages compared with the rectilinear k-t sampling pattern of echo-planar spectroscopic imaging, including being less demanding on gradient performance, but to date, it has not been widely adopted for clinical applications.

SENSE-encoded MRSI combines MRSI with reduced phaseencoding, multiple receiver coils, and "parallel" reconstruction techniques (as in parallel MR imaging) ${ }^{32}$ to reduce scan time. The 
basic principle of the parallel imaging technique is to use the inhomogeneous $B_{1}$ fields of multiple, phased-array coils to encode some of the spatial information, thereby allowing fewer conventional phase-encoding steps to be used, hence reducing scan time. In SENSE-encoded MRSI, Fourier transformation of undersampled $k$-space data leads to aliased spectroscopic images from each receiver channel, which can then be unfolded and reconstructed by using the sensitivity profile of each coil to produce a single spectroscopic image with uniform sensitivity. An attractive feature of sensitivity encoding is that it can be combined with any existing MRSI pulse sequence, so that there are no pulse sequence-related SNR losses, unlike those that can potentially occur with other fast MRSI methods. ${ }^{33} 2 \mathrm{D}$ and 3D MRSI involve phase-encoding in multiple directions, so SENSE-encoding can also be performed in 2D or 3D (provided that receive arrays with appropriate geometry are available), leading to large scan time reductions. $^{34}$

\section{Spectral Editing Techniques}

Although spectral editing can be performed in a number of different ways, currently the Mescher-Garwood (MEGA)-PRESS is the most widely used method, usually for the measurement of GABA at 3T. MEGA-PRESS combines PRESS localization with 2 frequency-selective editing pulses (which can also act to suppress water signal), ${ }^{35}$ which are alternately turned "on" and "off" in an interleaved fashion throughout the acquisition. For the detection of GABA, usually the frequency-selective editing pulse is placed at 1.9 ppm in the "on" (even) acquisition, and the "off" acquisition actually applies the pulse symmetrically on the opposite side of the water peak ( $4.7 \mathrm{ppm})$ at $7.5 \mathrm{ppm}$. Because the GABA resonance at $1.9 \mathrm{ppm}$ is coupled to the 3.0-ppm resonance, the resulting edited spectrum (derived from the difference between the "on" and "off" spectra) shows a peak at $3.0 \mathrm{ppm}$, while overlapping resonances are suppressed.

Other, less frequently used "editing" techniques for coupled molecules, include the 2D J-PRESS method, ${ }^{36,37}$ chemical shift selective filter, ${ }^{38}$ constant time PRESS, ${ }^{39}$ TE-averaged PRESS, ${ }^{40}$ and maximum-echo sampling methods. ${ }^{37}$ Some of these methods may also be combined with MRSI, for instance a 2D MRSI TEaveraged PRESS technique was used to generate Glu maps. ${ }^{41}$ Recently, with the advent of techniques for speeding up MRSI acquisitions such as compressed sensing, it has become possible to perform multidimensional MRSI scans (2 spectral, 3 spatial), which have promise for mapping compounds that are difficult to observe with conventional MRSI techniques. ${ }^{42}$

Currently, nearly all the techniques mentioned above, other than STEAM, PRESS, and conventional MRSI, are not commercially available and therefore are only performed by research groups with access to these methodologies.

\section{High-Field MR Spectroscopy}

Spectral SNR and chemical shift resolution increase with increasing magnetic field strength $\left(B_{0}\right)$, though the SNR increase may sometimes be less than the linear improvement predicted by theory. ${ }^{43}$ This outcome is most likely explained by the increase in spectral line widths with increasing $\mathrm{B}_{0}$. While field homogeneity is usually measured from voxel water line widths, typical metabolite (eg, for $\mathrm{Cr}$ or NAA) line widths in the human brain are $3.5 \mathrm{~Hz}$ at 1.5T, $5.5 \mathrm{~Hz}$ at $4 \mathrm{~T}$, and $9.5 \mathrm{~Hz}$ at 7T. ${ }^{44}$ The metabolite line width also depends on $\mathrm{B}_{0}$ field homogeneity and metabolite $\mathrm{T}_{2}$ relaxation time. It has been found that metabolite $\mathrm{T}_{2}$ relaxation times measured in vivo decrease with increasing $\mathrm{B}_{0} \cdot{ }^{45}$ For instance, the $\mathrm{T}_{2}$ of NAA drops from approximately $300-450 \mathrm{~ms}$ at $1.5 \mathrm{~T}^{43,46,47}$ to $210-300 \mathrm{~ms}$ at $3 \mathrm{~T},{ }^{43,48}$ to $185-230 \mathrm{~ms}$ at $4 \mathrm{~T},{ }^{45}$ and $140 \mathrm{~ms}$ at 7T. ${ }^{44}$ Therefore, high-field MR spectroscopy is best performed at short TEs (such as $\leq 35 \mathrm{~ms}$ ). Despite increasing line width, improved SNR and chemical resolution of spectra are shown at 7T compared with 3T, and 3T compared with 1.5T. ${ }^{43,44}$ Moreover, resonances from coupled spin systems such as GABA, Glu, and glutamine are better demonstrated at high field. Therefore, performing MR spectroscopy studies at the highest field available is recommended.

\section{Spectroscopic Applications in $\mathrm{MCl}$ and $\mathrm{AD}$}

$\mathrm{AD}$ is the most common cause of dementia; MCI is the transitional state between normal aging and $\mathrm{AD}$. The articles discussed below were the result of a PubMed search by using the phrases "Alzheimer disease or mild cognitive impairment" and "MR spectroscopy." The search was restricted to the English language (181 results). The articles discussed are classified into the following categories: diagnosis, differential diagnosis, therapy, and other applications.

\section{Diagnosis}

The Table shows methodologic details and results of studies that compared $\mathrm{AD}, \mathrm{MCI}$, and healthy controls. Reduction of NAA levels compared with age-matched healthy controls was the most frequent ${ }^{1} \mathrm{H}$-MRS finding in $\mathrm{AD} .{ }^{49,50}$ Reduced NAA/Cr ratios have been shown in the posterior cingulate gyrus, mesial temporal lobe, occipital lobe, parietal lobe, and frontal lobe. ${ }^{51-62}$ The "absolute" concentration of NAA was also decreased in the parietal lobe, occipital lobe, mesial temporal lobe, frontoparietal region, and hippocampus. ${ }^{63-69}$ These results are consistent with in vitro studies performed on postmortem $\mathrm{AD}$ brains, which showed that NAA decreases correlated with the severity of neuropathologic findings, such as amyloid plaques, neurofibrillary tangles, and the presence of apolipoprotein E genetic markers. ${ }^{60}$ Therefore, reduction of NAA levels might reflect either a loss of the neuronal cells or neuronal function, or both.

Increases of $\mathrm{mI}$ have also been reported in several anatomic locations in $\mathrm{AD}$, indicative of increased glial cell content. Increased $\mathrm{mI}$ has been reported most often in the posterior cingulate gyrus, temporal-parietal area, parietal white matter and occipital lobes. ${ }^{51,60,61,65,70}$ However, 2 studies reported no significant differences in $\mathrm{mI}$ levels between patients with $\mathrm{AD}$ and healthy controls. ${ }^{55,71}$ Some researchers have used the ratio of NAA/mI to increase the sensitivity of ${ }^{1} \mathrm{H}-\mathrm{MR}$ spectroscopy to metabolite changes in $\mathrm{AD} .{ }^{61,72,73}$ The NAA/mI ratio has been shown to be the more accurate MR spectroscopic measurement to differentiate patients with $\mathrm{AD}$ from healthy elderly.

There are conflicting reports on the TMA level in patients with AD. Some studies showed elevated TMA levels in $\mathrm{AD},{ }^{61,74-76}$ while others report decreased TMA levels ${ }^{6,69,77}$ or no change. ${ }^{53,60,62}$ It has been suggested that these disparate findings may be the result of 


\begin{tabular}{|c|c|c|c|c|c|}
\hline Reference & Method & Field $(T)$ & Region & Application & Finding ( $A D$ versus $N C$ ) \\
\hline Watanabe et al ${ }^{69}$ & PRESS & 1.5 & Hippocampus, PCG, occipital lobe & $\mathrm{AD}, \mathrm{MCl}, \mathrm{NC}$ & $\mathrm{NAA} \downarrow \mathrm{ml} \uparrow \mathrm{TMA} \downarrow \mathrm{tCr} \downarrow \star$ \\
\hline Kantarci et al ${ }^{60}$ & PRESS & 1.5 & PCG & $A D$, postmortem & $\mathrm{NAA} \downarrow \mathrm{ml} \uparrow \mathrm{TMA} \rightarrow$ \\
\hline Block et $\mathrm{al}^{53}$ & 2D-MRSI & 1.5 & $\begin{array}{l}\text { Hippocampus, temporal lobe, } \\
\text { occipital lobe }\end{array}$ & $A D, N C$ & $\mathrm{NAA} \downarrow \mathrm{TMA} \rightarrow$ \\
\hline Kantarci et $\mathrm{al}^{61}$ & PRESS & $1.5,3.0$ & PCG & $\mathrm{AD}, \mathrm{MCl}$ & $\mathrm{NAA} \downarrow \mathrm{ml} \uparrow \mathrm{TMA} \uparrow \mathrm{Glx} \rightarrow$ \\
\hline Chantal et $\mathrm{al}^{54}$ & PRESS & 1.5 & $\begin{array}{l}\text { MTL, prefrontal cortex, and } \\
\text { parietotemporal }\end{array}$ & $A D, N C$ & $\mathrm{NAA} \downarrow \mathrm{TMA} \downarrow \mathrm{ml} \uparrow \star$ \\
\hline Hattori et $\mathrm{al}^{58}$ & PRESS & 3.0 & PCG, parieto-occipital white matter & $\mathrm{AD}, \mathrm{NC}$ & NAA $\downarrow$ Glx $\downarrow$ \\
\hline Jessen et $\mathrm{al}^{67}$ & PRESS & 1.5 & MTL & $A D, N C$ & $\mathrm{NAA} \downarrow \mathrm{TMA} \rightarrow \mathrm{tCr} \rightarrow \star$ \\
\hline Garcia Santos et a $\left.\right|^{55}$ & PRESS & 1.5 & PCG & $\mathrm{AD}, \mathrm{MCl}, \mathrm{NC}$ & $\mathrm{NAA} \downarrow \mathrm{TMA} \rightarrow \mathrm{ml} \rightarrow$ \\
\hline Hancu et $\mathrm{al}^{57}$ & J-PRESS & 3.0 & PCG & $\mathrm{AD}, \mathrm{NC}$ & NAA $\downarrow$ Glu $\rightarrow$ \\
\hline Dixon et $\mathrm{al}^{63}$ & STEAM & 2.0 & Hippocampus & $A D, N C$ & $\mathrm{NAA} \downarrow \star$ \\
\hline Schott et $\mathrm{al}^{62}$ & PRESS & 1.5 & PCG & $A D, N C$ & $\mathrm{NAA} \downarrow \mathrm{ml} \uparrow \mathrm{TMA} \rightarrow$ \\
\hline Antuono et $\mathrm{al}^{52}$ & PRESS & 0.5 & PCG & $A D, N C$ & $\mathrm{NAA} \downarrow \mathrm{ml} \uparrow \mathrm{TMA} \rightarrow \mathrm{Glx} \downarrow$ \\
\hline Godbolt et al ${ }^{56}$ & PRESS & 1.5 & PCG & $A D, N C$ & $\mathrm{NAA} \downarrow \mathrm{ml} \uparrow$ \\
\hline Jessen et al ${ }^{66}$ & PRESS & 1.5 & MTL & $\mathrm{AD}, \mathrm{MCl}, \mathrm{NC}$ & $\mathrm{NAA} \downarrow \mathrm{TMA} \downarrow \mathrm{tCr} \downarrow \star$ \\
\hline Siger et $\mathrm{al}^{70}$ & 2D-MRSI & 1.5 & Frontal WM, parietal WM & $\mathrm{AD}, \mathrm{MCl}, \mathrm{NC}$ & $\mathrm{NAA} \downarrow \mathrm{ml} \uparrow$ \\
\hline Ackl et $\mathrm{al}^{51}$ & PRESS & 1.5 & Parietal WM, parietal GM, hippocampus & $\mathrm{AD}, \mathrm{MCl}, \mathrm{NC}$ & $\mathrm{NAA} \downarrow \mathrm{ml} \uparrow$ \\
\hline Falini et $\mathrm{al}^{64}$ & WBNAA & 1.5 & Whole brain & $\mathrm{AD}, \mathrm{MCl}, \mathrm{NC}$ & $\mathrm{NAA} \downarrow \star$ \\
\hline Frederick et $\mathrm{al}^{71}$ & PRESS & 1.5 & Temporal lobe & $\mathrm{AD}, \mathrm{MCl}, \mathrm{NC}$ & $\mathrm{NAA} \downarrow \mathrm{ml} \rightarrow \mathrm{TMA} \rightarrow$ \\
\hline Christiansen et $\mathrm{al}^{93}$ & STEAM & 1.5 & Hippocampus & $\mathrm{AD}, \mathrm{NC}$ & T2 values $\downarrow$ \\
\hline Chantal et $\mathrm{al}^{77}$ & PRESS & 1.5 & $\begin{array}{l}\text { MTL, prefrontal cortex, and } \\
\text { parietotemporal }\end{array}$ & $\mathrm{AD}, \mathrm{MCl}, \mathrm{NC}$ & $\mathrm{NAA} \downarrow \mathrm{TMA} \downarrow \mathrm{ml} \uparrow \star$ \\
\hline Huang et $\mathrm{al}^{65}$ & STEAM & 1.5 & Parietal lobe, occipital lobe & $\mathrm{AD}, \mathrm{NC}$ & $\mathrm{NAA} \downarrow \mathrm{ml} \uparrow \mathrm{TMA} \rightarrow \mathrm{tCr} \uparrow \star$ \\
\hline Schuff et al ${ }^{68}$ & 2D-MRSI & 1.5 & Frontoparietal region & $A D, N C$ & $\mathrm{NAA} \downarrow \mathrm{TMA} \rightarrow \mathrm{tCr} \rightarrow \star$ \\
\hline Parnetti et $\mathrm{al}^{72}$ & STEAM & 1.5 & Temporal GM, frontal WM & $A D, N C$ & $\mathrm{NAA} \downarrow \mathrm{TMA} \rightarrow \mathrm{ml} \uparrow \mathrm{tCr} \rightarrow \star$ \\
\hline Frederick et $\mathrm{al}^{94}$ & STEAM & 1.5 & Parietal lobe, temporal lobe & $A D, N C$ & $\mathrm{NAA} \downarrow$ TMA $\rightarrow$ \\
\hline Jessen et $\mathrm{al}^{59}$ & PRESS & 1.5 & Parietal GM, MTL & $A D, N C$ & $\mathrm{NAA} \downarrow \mathrm{TMA} \downarrow$ \\
\hline Rose et $\mathrm{al}^{73}$ & STEAM & 2.0 & Parietal midline & $A D, N C$ & $\mathrm{NAA} \downarrow \mathrm{ml} \uparrow \mathrm{TMA} \rightarrow \mathrm{tCr} \rightarrow$ \\
\hline Kantarci et $\mathrm{al}^{74}$ & PRESS & 1.5 & PCG, temporal lobe, occipital lobe & $\mathrm{AD}, \mathrm{MCl}, \mathrm{NC}$ & $\mathrm{NAA} \downarrow \mathrm{ml} \uparrow \mathrm{TMA} \uparrow$ \\
\hline Haley et $\mathrm{al}^{95}$ & STEAM & 1.5 & Frontal WM & $\mathrm{AD}, \mathrm{NC}$ & $\mathrm{NAA} \downarrow \mathrm{TMA} \rightarrow$ \\
\hline Mackay et $\mathrm{al}^{75}$ & 2D-MRSI & 2.0 & GM, WM & $A D, N C$ & $\mathrm{NAA} \downarrow \downarrow \mathrm{TMA} \uparrow$ \\
\hline Schuff et $\mathrm{al}^{76}$ & 2D-MRSI & 1.5 & Hippocampus & $A D, N C$ & $\mathrm{NAA} \downarrow \mathrm{ml} \uparrow \mathrm{TMA} \uparrow$ \\
\hline
\end{tabular}

Note:-NC indicates healthy control; PRESS-J, TE-averaged PRESS; MTL, mesial temporal lobe; WBNAA, Whole-brain NAA; $\uparrow$, significantly increased; $\downarrow$, significantly decreased; $\rightarrow$, no significant differences; NC, normal controls; PCG, posterior cingulate gyrus.

${ }^{a}$ Metabolite concentrations are ratios to $\mathrm{tCr}$, unless a star indicates that the metabolite level has been calculated by reference to the water signal instead.

possible apolipoprotein $E$ allele effects on membrane metabolism or breakdown, differences in MR spectroscopy methods (in particular TE), or variations in anatomic voxel placement. ${ }^{68}$

While the tCr peak is generally thought to be stable in $\mathrm{AD}$, some studies have demonstrated decreased tCr levels in patients with $\mathrm{AD}$ versus healthy controls in the occipital lobe ${ }^{69}$ and increased tCr levels in the parietal and occipital lobes. ${ }^{65}$

The Glx peak has only been investigated in a few studies; these have mostly reported reduced Glx levels in patients with AD compared with controls in the posterior cingulate gyrus and parietooccipital white matter, ${ }^{58}$ though 1 study reported no difference in Glx between patients with $\mathrm{AD}$ and healthy controls. ${ }^{61}$

Finally, 1 study demonstrated decreased levels of glutathione in the right frontal cortex of female patients with $\mathrm{AD}$ compared with healthy female controls and decreased glutathione levels in the left frontal cortex of male patients with $\mathrm{AD} .{ }^{78}$ In this study, the glutathione level also showed a trend toward reduction in patients with MCI compared with healthy subjects, though the difference was not statistically significant. ${ }^{78}$

Generally, metabolic differences between patients with $\mathrm{AD}$, MCI, and controls are fairly small, and appreciable scatter (overlap) exists between groups. Therefore, MR spectroscopy in isolation usually cannot be used in individual subjects for the diagnosis of AD. Metabolite concentrations should be corrected for CSF contamination because brain atrophy is typically very significant in both the elderly healthy control population and particularly in patients with $\mathrm{AD}$.

\section{Differential Diagnosis}

A number of studies have compared subcortical ischemic vascular dementia with $\mathrm{AD} . \mathrm{mI} / \mathrm{tCr}$ was found to be higher, and $\mathrm{tNAA} / \mathrm{tCr}$, lower, in patients with $\mathrm{AD}$ compared with those with subcortical ischemic vascular dementia. ${ }^{79,80}$ It has been reported that there is a significant correlation between Mini-Mental State Examination score and $\mathrm{tNAA} / \mathrm{mI}$ and $\mathrm{tNAA} / \mathrm{tCr}$ in patients with $\mathrm{AD}$ but that patients with subcortical ischemic vascular dementia showed no correlations. ${ }^{81}$ In 1 study of $\mathrm{AD}$ and frontotemporal dementia, $\mathrm{tNAA} / \mathrm{tCr}$ was reduced in the posterior cingulate gyrus in both patients with $\mathrm{AD}$ and frontotemporal dementia; however, the patients with $\mathrm{AD}$ showed a posterior dominant decrease, whereas there was a frontal predominant decrease in the patients with frontotemporal dementia, ${ }^{82}$ while another study found no significant metabolic differences between $\mathrm{AD}$ and frontotemporal dementia in the posterior cingulate gyrus. ${ }^{79}$ However, it was found that $\mathrm{tNAA} / \mathrm{tCR}$ was higher in patients with dementia with Lewy bodies than $\mathrm{AD}$ in the posterior cingulate gyrus. ${ }^{79}$ Finally, 1 study found that MR spectroscopy can differentiate between $\mathrm{AD}$ and $\mathrm{MCI}$, with the strongest effect seen with the tNAA/tCr ratio in the 
left occipital cortex, but it could not differentiate between different types of MCI categorized according to the suspected underlying pathology (eg, neurodegeneration, vascular, or dysphoric or dysthymic disorders). ${ }^{83}$

\section{Effects of Therapy}

A number of studies have investigated the effect of the acetylcholinesterase inhibitor donepezil in $\mathrm{AD} .{ }^{84-87}$ It was found that $\mathrm{tNAA}$ and $\mathrm{tNAA} / \mathrm{tCr}$ tended to be higher in the donepezil-treated patients compared with a placebo, ${ }^{86}$ although 1 study found that tNAA, TMA, tNAA/tCr, TMA/tCr, and mI/tCr were all decreased in patients with $\mathrm{AD}$ after treatment. ${ }^{84}$ An MRSI study of the muscarinic acetylcholine receptor agonist xanomeline found no metabolic differences before or after treatment in either study drug or placebo groups but did find a positive correlation between parietal lobe gray matter TMA/tCr ratio and cognitive performance. ${ }^{88}$ Finally, in a small trial $(n=10)$ of the alkaloid galantamine, levels of Glu and Glu/tCr in the right hippocampus increased after 4 months of treatment, and these changes were associated with increased cognitive performance. ${ }^{89}$

\section{Other Applications}

One study of MCI and patients with early AD found correlations between various cognitive measures (eg, verbal learning performance, memory) and cortical metabolite ratios. ${ }^{90}$ Another compared MR spectroscopy and diffusion tensor imaging findings and found a positive correlation between $\mathrm{mI} / \mathrm{tCr}$ and fractional anisotropy values and a negative correlation between $\mathrm{tNAA} / \mathrm{tCr}$ and mean diffusivity values in patients with AD. ${ }^{91}$ Finally, in a study of 15 control subjects and 22 patients with $\mathrm{AD}$, it was reported that correlations exist between elevated basal ganglia TMA signal and poorer performance on learning tasks, with more significant findings in male patients with $\mathrm{AD}$, suggesting sex-specific effects. ${ }^{92}$

\section{CONCLUSIONS}

Although an abundance of studies have shown metabolic changes in the brain in subjects with MCI and $\mathrm{AD}$, at present, MR spectroscopy is little used in the clinical evaluation of subjects with dementia. This is probably due to several reasons, including lack of standardized methodology, overlap of spectral patterns between different pathologies (ie, relative lack of specificity), lack of reimbursement, and lack of treatment options in most dementias. Arguably the most important role for MR spectroscopy may be in predicting the development of dementia in patients with MCI, information that is highly valued by patients and their families. The relative value of MR spectroscopy, performed with the best available contemporary methods, needs further evaluation in this regard; its performance should be compared with other imaging measures from MR imaging and positron-emission tomography, in particular amyloid imaging methods, as well as other biomarkers and clinical testing. For the time being, MR spectroscopy may find a greater role as a surrogate marker of brain metabolic health in clinical trials, perhaps both for patient selection and monitoring outcome.
Disclosures: Peter B. Barker-UNRELATED: Consultancy: I am a consultant for Olea Medical for the development of a commercial MR spectroscopy data analysis software package.

\section{REFERENCES}

1. Mueller SG, Schuff N, Weiner MW. Evaluation of treatment effects in Alzheimer's and other neurodegenerative diseases by MRI and MRS. NMR Biomed 2006;19:655-68

2. Barker $P B$, Lin $D D$. In vivo proton MR spectroscopy of the human brain. Prog Nucl Magn Reson Spectrosc 2006;49:99-128

3. Valenzuela MJ, Sachdev P. Magnetic resonance spectroscopy in AD. Neurology 2001;56:592-98

4. Simmons ML, Frondoza CG, Coyle JT. Immunocytochemical localization of $\mathrm{N}$-acetyl-aspartate with monoclonal antibodies. Neuroscience 1991;45:37-45

5. Urenjak J, Williams SR, Gadian DG, et al. Specific expression of $\mathrm{N}$-acetylaspartate in neurons, oligodendrocyte-type-2 astrocyte progenitors, and immature oligodendrocytes in vitro. J Neurochem 1992;59:55-61

6. De Stefano N, Matthews PM, Arnold DL. Reversible decreases in $\mathrm{N}$-acetylaspartate after acute brain injury. Magn Reson Med 1995;34:721-27

7. Mader I, Roser W, Kappos L, et al. Serial proton MR spectroscopy of contrast-enhancing multiple sclerosis plaques: absolute metabolic values over 2 years during a clinical pharmacological study. AJNR Am J Neuroradiol 2000;21:1220-27

8. Pettegrew JW, McClure RJ, Panchalingam K. Spectroscopic imaging of schizophrenia. In: Shenton ME and Turetsky BI, eds. Understanding Neupsychiatric Disorders: Insights from Neuroimaging. New York: Cambridge University Press; 2011:48-77

9. Loos C, Achten E, Santens P. Proton magnetic resonance spectroscopy in Alzheimer's disease: a review. Acta Neurol Belg 2010;110: 291-98

10. Gill SS, Small RK, Thomas DG, et al. Brain metabolites as 1 H NMR markers of neuronal and glial disorders. NMR Biomed 1989;2: 196-200

11. Pfefferbaum A, Adalsteinsson E, Spielman D, et al. In vivo spectroscopic quantification of the $\mathrm{N}$-acetyl moiety, creatine, and choline from large volumes of brain gray and white matter: effects of normal aging. Magn Reson Med 1999;41:276-84

12. Ross BD. Biochemical considerations in $\mathbf{1 H}$ spectroscopy: glutamate and glutamine; myo-inositol and related metabolites. NMR Biomed 1991;4:59-63

13. Brand A, Richter-Landsberg C, Leibfritz D. Multinuclear NMR studies on the energy metabolism of glial and neuronal cells. Dev Neurosci 1993;15:289-98

14. Flögel U, Willker W, Leibfritz D. Regulation of intracellular $\mathbf{p H}$ in neuronal and glial tumour cells, studied by multinuclear NMR spectroscopy. NMR Biomed 1994;7:157-66

15. Lazeyras F, Charles HC, Tupler LA, et al. Metabolic brain mapping in Alzheimer's disease using proton magnetic resonance spectroscopy. Psychiatry Res 1998;82:95-106

16. Kreis R, Ross BD, Farrow NA, et al. Metabolic disorders of the brain in chronic hepatic encephalopathy detected with H-1 MR spectroscopy. Radiology 1992;182:19-27

17. McCormick D. GABA as an inhibitory neurotransmitter in human cerebral cortex. J Neurophysiol 1989;62:1018-27

18. Govindaraju V, Young K, Maudsley AA. Proton NMR chemical shifts and coupling constants for brain metabolites. NMR Biomed 2000;13:129-53

19. Bottomley PA, inventor. Selective volume method for performing localized NMR spectroscopy. US patent 4480228 1984. October 30, 1984

20. Frahm J, Bruhn H, Gyngell ML, et al. Localized high-resolution proton NMR spectroscopy using stimulated echoes: initial applications to human brain in vivo. Magn Reson Med 1989;9:79-93

21. Collins CM, Liu W, Schreiber W, et al. Central brightening due to 
constructive interference with, without, and despite dielectric resonance. J Magn Reson Imaging 2005;21:192-96

22. Garwood M, DelaBarre L. The return of the frequency sweep: designing adiabatic pulses for contemporary NMR. J Magn Reson 2001;153:155-77

23. Scheenen TW, Heerschap A, Klomp DW. Towards 1H-MRSI of the human brain at $7 \mathrm{~T}$ with slice-selective adiabatic refocusing pulses. MAGMA 2008;21:95-101

24. Scheenen TW, Klomp DW, Wijnen JP, et al. Short echo time 1HMRSI of the human brain at 3T with minimal chemical shift displacement errors using adiabatic refocusing pulses. Magn Reson Med 2008;59:1-6

25. Zhu H, Barker PB. MR spectroscopy and spectroscopic imaging of the brain. Methods Mol Biol 2011;711:203-26

26. Mekle R, Mlynarik V, Gambarota G, et al. MR spectroscopy of the human brain with enhanced signal intensity at ultrashort echo times on a clinical platform at 3T and 7T. Magn Reson Med 2009;61:1279-85

27. Duyn JH, Gillen J, Sobering G, et al. Multisection proton MR spectroscopic imaging of the brain. Radiology 1993;188:277-82

28. Spielman D, Meyer C, Macovski A, et al. 1H spectroscopic imaging using a spectral-spatial excitation pulse. Magn Reson Med 1991; 18:269-79

29. Posse $\mathrm{S}$, Tedeschi G, Risinger R, et al. High speed $1 \mathrm{H}$ spectroscopic imaging in human brain by echo planar spatial-spectral encoding. Magn Reson Med 1995;33:34-40

30. Ebel A, Soher BJ, Maudsley AA. Assessment of 3D proton MR echoplanar spectroscopic imaging using automated spectral analysis. Magn Reson Med 2001;46:1072-78

31. Adalsteinsson E, Irarrazabal P, Spielman DM, et al. Three-dimensional spectroscopic imaging with time-varying gradients. Magn Reson Med 1995;33:461-66

32. Pruessmann KP, Weiger M, Scheidegger MB, et al. SENSE: sensitivity encoding for fast MRI. Magn Reson Med 1999;42:952-62

33. Pohmann R, von Kienlin M, Haase A. Theoretical evaluation and comparison of fast chemical shift imaging methods. J Magn Reson 1997;129:145-60

34. Dydak U, Weiger M, Pruessmann KP, et al. Sensitivity-encoded spectroscopic imaging. Magn Reson Med 2001;46:713-22

35. Mescher M, Merkle H, Kirsch J, et al. Simultaneous in vivo spectral editing and water suppression. NMR Biomed 1998;11:266-72

36. Ryner LN, Sorenson JA, Thomas MA. Localized 2D J-resolved $1 \mathrm{H}$ MR spectroscopy: strong coupling effects in vitro and in vivo. Magn Reson Imaging 1995; 13:853-69

37. Schulte RF, Lange T, Beck J, et al. Improved two-dimensional J-resolved spectroscopy. NMR Biomed 2006;19:264-70

38. Schulte RF, Trabesinger AH, Boesiger P. Chemical-shift-selective filter for the in vivo detection of J-coupled metabolites at 3T. Magn Reson Med 2005;53:275-81

39. Mayer D, Spielman DM. Detection of glutamate in the human brain at $3 \mathrm{~T}$ using optimized constant time point resolved spectroscopy. Magn Reson Med 2005;54:439-42

40. Hurd R, Sailasuta N, Srinivasan R, et al. Measurement of brain glutamate using TE-averaged PRESS at 3T. Magn Reson Med 2004;51:435-40

41. Srinivasan R, Cunningham C, Chen A, et al. TE-averaged two-dimensional proton spectroscopic imaging of glutamate at $3 \mathrm{~T}$. Neuroimage 2006;30:1171-78

42. Furuyama JK, Wilson NE, Burns BL, et al. Application of compressed sensing to multidimensional spectroscopic imaging in human prostate. Magn Reson Med 2012;67:1499-505

43. Barker PB, Hearshen DO, Boska MD. Single-voxel proton MRS of the human brain at 1.5T and 3.0T. Magn Reson Med 2001;45:765-69

44. Tkác I, Andersen P, Adriany G, et al. In vivo $1 \mathrm{H}$ NMR spectroscopy of the human brain at 7 T. Magn Reson Med 2001;46:451-56

45. Posse S, Cuenod CA, Risinger R, et al. Anomalous transverse relaxation in $1 \mathrm{H}$ spectroscopy in human brain at $\mathbf{4}$ Tesla. Magn Reson Med 1995;33:246-52
46. Brief EE, Whittall KP, Li DK, et al. Proton T2 relaxation of cerebral metabolites of normal human brain over large TE range. $N M R$ Biomed 2005; 18:14-18

47. Kreis R, Ernst T, Ross BD. Development of the human brain: in vivo quantification of metabolite and water content with proton magnetic resonance spectroscopy. Magn Reson Med 1993;30:424-37

48. Mlynárik V, Gruber S, Moser E. Proton T (1) and T (2) relaxation times of human brain metabolites at 3 Tesla. NMR Biomed 2001;14:325-31

49. Graff-Radford J, Kantarci K. Magnetic resonance spectroscopy in Alzheimer's disease. Neuropsychiatr Dis Treat 2013;9:687-96

50. Mandal PK. Magnetic resonance spectroscopy (MRS) and its application in Alzheimer's disease. Concepts in Magnetic Resonance Part A 2007;30A:40-64

51. Ackl N, Ising M, Schreiber YA, et al. Hippocampal metabolic abnormalities in mild cognitive impairment and Alzheimer's disease. Neurosci Lett 2005;384:23-28

52. Antuono PG, Jones JL, Wang Y, et al. Decreased glutamate + glutamine in Alzheimer's disease detected in vivo with (1)H-MRS at 0.5 T. Neurology 2001;56:737-42

53. Block W, Jessen F, Traber F, et al. Regional N-acetylaspartate reduction in the hippocampus detected with fast proton magnetic resonance spectroscopic imaging in patients with Alzheimer disease. Arch Neurol 2002;59:828-34

54. Chantal S, Labelle M, Bouchard RW, et al. Correlation of regional proton magnetic resonance spectroscopic metabolic changes with cognitive deficits in mild Alzheimer disease. Arch Neurol 2002; 59:955-62

55. Garcia Santos JM, Gavrila D, Antúnez C, et al. Magnetic resonance spectroscopy performance for detection of dementia, Alzheimer's disease and mild cognitive impairment in a community-based survey. Dement Geriatr Cogn Disord 2008;26:15-25

56. Godbolt AK, Waldman AD, MacManus DG, et al. MRS shows abnormalities before symptoms in familial Alzheimer disease. Neurology 2006;66:718-22

57. Hancu I, Zimmerman EA, Sailasuta N, et al. 1H MR spectroscopy using TE averaged PRESS: a more sensitive technique to detect neurodegeneration associated with Alzheimer's disease. Magn Reson Med 2005;53:777-82

58. Hattori N, Abe K, Sakoda S, et al. Proton MR spectroscopic study at 3 Tesla on glutamate/glutamine in Alzheimer's disease. Neuroreport 2002;13:183-86

59. Jessen F, Block W, Traber F, et al. Proton MR spectroscopy detects a relative decrease of $\mathrm{N}$-acetylaspartate in the medial temporal lobe of patients with AD. Neurology 2000;55:684-88

60. Kantarci K, Knopman DS, Dickson DW, et al. Alzheimer disease: postmortem neuropathologic correlates of antemortem $1 \mathrm{H}$ MR spectroscopy metabolite measurements. Radiology 2008;248:210-20

61. Kantarci K, Reynolds G, Petersen RC, et al. Proton MR spectroscopy in mild cognitive impairment and Alzheimer disease: comparison of 1.5 and 3 T. AJNR Am J Neuroradiol 2003;24:843-49

62. Schott JM, Frost C, MacManus DG, et al. Short echo time proton magnetic resonance spectroscopy in Alzheimer's disease: a longitudinal multiple time point study. Brain 2010;133:3315-22

63. Dixon RM, Bradley KM, Budge MM, et al. Longitudinal quantitative proton magnetic resonance spectroscopy of the hippocampus in Alzheimer's disease. Brain 2002;125:2332-41

64. Falini A, Bozzali M, Magnani G, et al. A whole brain MR spectroscopy study from patients with Alzheimer's disease and mild cognitive impairment. Neuroimage 2005;26:1159-63

65. Huang W, Alexander GE, Chang L, et al. Brain metabolite concentration and dementia severity in Alzheimer's disease: a (1)H MRS study. Neurology 2001;57:626-32

66. Jessen F, Gur O, Block W, et al. A multicenter (1)H-MRS study of the medial temporal lobe in AD and MCI. Neurology 2009;72:1735-40

67. Jessen F, Traeber F, Freymann N, et al. A comparative study of the 
different $\mathrm{N}$-acetylaspartate measures of the medial temporal lobe in Alzheimer's disease. Dement Geriatr Cogn Disord 2005;20:178-83

68. Schuff N, Amend DL, Meyerhoff DJ, et al. Alzheimer disease: quantitative H-1 MR spectroscopic imaging of frontoparietal brain. $\mathrm{Ra}$ diology 1998;207:91-102

69. Watanabe T, Shiino A, Akiguchi I. Absolute quantification in proton magnetic resonance spectroscopy is useful to differentiate amnesic mild cognitive impairment from Alzheimer's disease and healthy aging. Dement Geriatr Cogn Disord 2010;30:71-77

70. Siger M, Schuff N, Zhu X, et al. Regional myo-inositol concentration in mild cognitive impairment using $1 \mathrm{H}$ magnetic resonance spectroscopic imaging. Alzheimer Dis Assoc Disord 2009;23:57-62

71. Frederick BD, Lyoo IK, Satlin A, et al. In vivo proton magnetic resonance spectroscopy of the temporal lobe in Alzheimer's disease. Prog Neuropsychopharmacol Biol Psychiatry 2004;28:1313-22

72. Parnetti L, Tarducci R, Presciutti O, et al. Proton magnetic resonance spectroscopy can differentiate Alzheimer's disease from normal aging. Mech Ageing Dev 1997;97:9-14

73. Rose SE, de Zubicaray GI, Wang D, et al. A 1H MRS study of probable Alzheimer's disease and normal aging: implications for longitudinal monitoring of dementia progression. Magn Reson Imaging 1999;17:291-99

74. Kantarci K, Jack CR Jr, Xu YC, et al. Regional metabolic patterns in mild cognitive impairment and Alzheimer's disease: a 1H MRS study. Neurology 2000;55:210-17

75. MacKay S, Meyerhoff DJ, Constans JM, et al. Regional gray and white matter metabolite differences in subjects with $\mathrm{AD}$, with subcortical ischemic vascular dementia, and elderly controls with $1 \mathrm{H}$ magnetic resonance spectroscopic imaging. Arch Neurol 1996;53:167-74

76. Schuff N, Amend D, Ezekiel F, et al. Changes of hippocampal Nacetyl aspartate and volume in Alzheimer's disease: a proton MR spectroscopic imaging and MRI study. Neurology 1997;49:1513-21

77. Chantal S, Braun CM, Bouchard RW, et al. Similar 1H magnetic resonance spectroscopic metabolic pattern in the medial temporal lobes of patients with mild cognitive impairment and Alzheimer disease. Brain Res 2004;1003:26-35

78. Mandal PK, Tripathi M, Sugunan S. Brain oxidative stress: detection and mapping of anti-oxidant marker 'glutathione' in different brain regions of healthy male/female, $\mathrm{MCI}$ and Alzheimer patients using non-invasive magnetic resonance spectroscopy. Biochem Biophys Res Commun 2012;417:43-48

79. Kantarci K, Petersen RC, Boeve BF, et al. 1H MR spectroscopy in common dementias. Neurology 2004;63:1393-98

80. Weiss U, Bacher R, Vonbank H, et al. Cognitive impairment: assessment with brain magnetic resonance imaging and proton magnetic resonance spectroscopy. J Clin Psychiatry 2003;64:235-42

81. Waldman AD, Rai GS. The relationship between cognitive impairment and in vivo metabolite ratios in patients with clinical Alzhei- mer's disease and vascular dementia: a proton magnetic resonance spectroscopy study. Neuroradiology 2003;45:507-12

82. Mihara M, Hattori N, Abe K, et al. Magnetic resonance spectroscopic study of Alzheimer's disease and frontotemporal dementia/ Pick complex. Neuroreport 2006;17:413-16

83. Fayed N, Davila J, Oliveros A, et al. Utility of different MR modalities in mild cognitive impairment and its use as a predictor of conversion to probable dementia. Acad Radiol 2008;15:1089-98

84. Bartha R, Smith M, Rupsingh R, et al. High field (1)H MRS of the hippocampus after donepezil treatment in Alzheimer disease. Prog Neuropsychopharmacol Biol Psychiatry 2008;32:786-93

85. Jessen F, Traeber F, Freymann K, et al. Treatment monitoring and response prediction with proton $\mathrm{MR}$ spectroscopy in $\mathrm{AD}$. Neurology 2006;67:528-30

86. Krishnan KR, Charles HC, Doraiswamy PM, et al. Randomized, placebo-controlled trial of the effects of donepezil on neuronal markers and hippocampal volumes in Alzheimer's disease. Am J Psychiatry 2003;160:2003-11

87. Modrego PJ, Fayed N, Errea JM, et al. Memantine versus donepezil in mild to moderate Alzheimer's disease: a randomized trial with magnetic resonance spectroscopy. Eur J Neurol 2010;17:405-12

88. Frederick B, Satlin A, Wald LL, et al. Brain proton magnetic resonance spectroscopy in Alzheimer disease: changes after treatment with xanomeline. Am J Geriatr Psychiatry 2002;10:81-88

89. Penner J, Rupsingh $\mathrm{R}$, Smith $\mathrm{M}$, et al. Increased glutamate in the hippocampus after galantamine treatment for Alzheimer disease. Prog Neuropsychopharmacol Biol Psychiatry 2010;34:104-10

90. Rami L, Gómez-Ansón B, Bosch B, et al. Cortical brain metabolism as measured by proton spectroscopy is related to memory performance in patients with amnestic mild cognitive impairment and Alzheimer's disease. Dement Geriatr Cogn Disord 2007;24:274-79

91. Ding B, Chen K-M, Ling HW, et al. Diffusion tensor imaging correlates with proton magnetic resonance spectroscopy in posterior cingulate region of patients with Alzheimer's disease. Dement Geriatr Cogn Disord 2008;25:218-25

92. Colla M, Ende G, Bohrer M, et al. MR spectroscopy in Alzheimer's disease: gender differences in probabilistic learning capacity. $\mathrm{Neu}$ robiol Aging 2003;24:545-52

93. Christiansen P, Schlosser A, Henriksen O. Reduced N-acetylaspartate content in the frontal part of the brain in patients with probable Alzheimer's disease. Magn Reson Imaging 1995;13:457-62

94. Frederick BB, Satlin A, Yurgelun-Todd DA, et al. In vivo proton magnetic resonance spectroscopy of Alzheimer's disease in the parietal and temporal lobes. Biol Psychiatry 1997;42:147-50

95. Haley AP, Knight-Scott J, Fuchs KL, et al. Shortening of hippocampal spin-spin relaxation time in probable Alzheimer's disease: a $1 \mathrm{H}$ magnetic resonance spectroscopy study. Neurosci Lett 2004;362: $167-70$ 